\title{
Detection of volatile organic compounds (VOCs) using an optical fibre long period grating with a calixarene anchored mesoporous thin film
}

\author{
S. Korposh ${ }^{1}$, F. Davis ${ }^{2}$, S. W. James ${ }^{1}$, T. Wang ${ }^{3}$ S.-W. Lee ${ }^{3}$, S. Higson ${ }^{2}$ and R. P. Tatam ${ }^{1}$ \\ ${ }^{1}$ Department of Engineering Photonics, School of Engineering, Cranfield University, Cranfield, \\ Bedfordshire MK43 0AL, UK \\ ${ }^{2}$ School of Health, Cranfield University, Cranfield, Bedfordshire MK43 0AL, UK \\ ${ }^{3}$ Department of Chemical Process and Environment, Graduate School of Environmental \\ Engineering, The University of Kitakyushu, Kitakyushu, 808-0135, Japan
}

\begin{abstract}
A long period grating (LPG) modified with a mesoporous film infused with a functional compound, calix[4]arene, was employed for the detection of volatile organic compounds (VOCs). The mesoporous film consisted of an inorganic part, of $\mathrm{SiO}_{2}$ nanoparticles (NPs) along with an organic moiety of poly(allylamine hydrochloride) polycation PAH, which was finally infused with functional compound, p-sulphanatocalix[4]arene (CA[4]). The LPG sensor was designed to operate at the phase matching turning point to provide the highest sensitivity. The sensing mechanism is based on the measurement of the refractive index (RI) change induced by the complexion of the VOCs with calix[4]arene (CA). The LPG modified with 5 cycles of $\left(\mathrm{SiO}_{2} \mathrm{NPs} / \mathrm{PAH}\right)_{5} \mathrm{PAA}$ responded to exposure to chloroform and benzene vapours. The sensitivity to humidity as an interfering parameter was also investigated.
\end{abstract}

Keywords: Long period grating (LPG), volatile organic compounds (VOCs), phase matching turning point (PMTP), mesoporous film, layer-by-layer (LbL), calix[4]arene (CA[4])

\section{INTRODUCTION}

Among the many classes of contaminants to be found within the indoor air, volatile organic compounds, VOCs, have been identified as a major cause for concern [1]. Total VOC concentration (TVOC)is generally used as an indicator of the potency of VOCs to cause health effects $\left[^{2}\right]$. Currently, measurements of the concentrations of gaseous contaminants at or near levels that are acceptable in terms of indoor air quality are not easily achieved. Expensive items of analytical equipment that facilitate techniques based on sampling and analysis are all that is available for TVOC measurement, and these must be calibrated and operated by experienced personnel. Consequently, the development of sensitive, cheap and reliable sensors capable of generating measurement results in real time is highly desirable.

Optical fibre long period gratings (LPGs) coated with chemically sensitive materials have emerged as a promising platform for fibre optic sensors [3, 4]. Typically, the transmission spectrum of an LPG contains a number of resonance bands, each corresponding to coupling to a different cladding mode and each showing a different sensitivity to environmental perturbation, which has been noted to offer the potential for multi-parameter sensing. The wavelengths at which light is coupled from the core to the cladding modes is governed by the phase matching equation

$$
\lambda_{x}=\left(n_{\text {core }}-n_{\text {clad }(x)}\right) \Lambda
$$

where $\lambda_{x}$ represents the wavelength at which light is coupled to the $\mathrm{LP}_{0 \mathrm{x}}$ cladding mode, $n_{\text {core }}$ is the effective refractive index of the mode propagating in the core of the fibre, $n_{\text {clad }(x)}$ is the effective index of the $\mathrm{LP}_{0 \mathrm{x}}$ cladding mode and $\Lambda$ is the period of the LPG. The dispersion of an optical fibre is such that the difference between the core and cladding mode effective indices exhibits a turning point where the value is maximum. For an FBG fabricated with a period such that equation (1) is satisfied at the phase matching turning point (PMTP), it has been shown that the sensitivity of the transmission spectrum to perturbation is at its maximum, and that for subsequent decreases in $\left(\mathrm{n}_{\text {core }}-\mathrm{n}_{\text {clad }(\mathrm{x})}\right)$, the LPGs transmission spectrum is characterised by the formation of a broad resonance band that subsequently splits into two.

Recently, LPGs modified with porous coatings have attracted a lot of interest. For instance, we have demonstrated a new approach to LPG based chemical sensing: the chemical infusion of analytes into a mesoporous coating that consisted of a multilayer film of $\mathrm{SiO}_{2}$ nanoparticles $\left(\mathrm{SiO}_{2} \mathrm{NPs}\right)$ deposited using the LbL technique [5]. In this study, the concept

Fifth European Workshop on Optical Fibre Sensors, edited by Leszek R. Jaroszewicz, Proc. of SPIE Vol. 8794, 87941 · C 2013 SPIE · CCC code: 0277-786X/13/\$18 · doi: 10.1117/12.2026270

Copyright 2013 Society of Photo Optical Instrumentation Engineers. One print or electronic copy may be made for personal use only.

Systematic reproduction and distribution, duplication of any material in this paper for a fee or for commercial purposes, or modification of the content of the paper are prohibited. 
explored in our previous work [3] is developed further to demonstrate a VOC-pecific chemical sensor. A novel 2-stage approach to the development of the sensor is explored. The first stage involves the deposition of the mesoporous coating onto the LPG. In the second stage a functional material, chosen to be sensitive to the analyte of interest, is infused into the base mesoporous coating. In this work the p-sulphanatocalix[4]arene (CA[4]) was used as a functional compound that has shape in the form of a cup with a well-defined cavity capable of host-guest interaction with various guest ions and small molecules, such as VOCs [6]. The LPG coated with mesoporous thin film infused with CA[4] was exposed to saturated vapour concentrations of benzene and chloroform. The response to water, a potential interfering measurand, was also investigated.

\section{EXPERIMENT}

\subsection{LPG fabrication and gas measurements}

LPGs of length $40 \mathrm{~mm}$ and with a grating period of $111.5 \mu \mathrm{m}$ were fabricated in boron-germanium co-doped optical fibre (Fibercore PS750) with cut-off wavelength $670 \mathrm{~nm}$ in a point-by-point fashion, side-illuminating the optical fibre by the output from a frequency-quadrupled Nd:YAG laser, operating at $266 \mathrm{~nm}$. The transmission spectrum of the optical fibre was recorded by coupling the output from a tungsten-halogen lamp (Ocean Optics HL-2000) into the fibre, analyzing the transmitted light using a fibre coupled CCD spectrometer (Ocean Optics HR4000). The grating period was selected such that the LPG operated at or near the phase matching turning point, which, for coupling to a particular cladding mode (in this case $\mathrm{LP}_{019}$ ), ensured optimised sensitivity [7]. $\mathrm{SiO}_{2}$ nanoparticles (NPs) were deposited onto the surface of the fibre using the LbL process, as schematically illustrated in Figure 1a. As the LPG transmission spectrum is known to be sensitive to bending, for the film deposition process and VOC detection experiments, the optical fibre containing the LPG was fixed within a special holder, such that the section of the fibre containing the LPG was taut and straight throughout the experiments. For film deposition, the section of the optical fibre containing the LPG, with its surface treated such that it was terminated with $\mathrm{OH}$ groups, was alternately immersed into a $0.13 \mathrm{wt} \%$ solution containing a positively charged polymer, $\mathrm{PAH}$, and, after washing, into a $1 \mathrm{wt} \%$ solution containing the negatively charged $\mathrm{SiO}_{2} \mathrm{NPs}$ solution, each for $15 \mathrm{~min}$, thereby forming mesoporous film on the LPG topical fibre, Figure $1 \mathrm{~b}$. This process was repeated until the required coating thickness was achieved. When the required film thickness had been achieved (i.e. when the development of the $\mathrm{LP}_{019}$ resonance band was observed with the fibre in air), after 5 deposition cycles, the coated fibre was immersed in a $1 \mathrm{mM}$ aqueous solution of $\mathrm{CA}[4]$ as functional compound for $2 \mathrm{~h}$, which was infused into the porous coating and provided the sensor with its specificity. Due to the electronegative sulfonic groups present in the $\mathrm{CA}[4]$ compound, Figure 1a, electrostatic interactions occur between $\mathrm{CA}[4]$ and cationic $\mathrm{PAH}$ in the $\mathrm{PAH} / \mathrm{SiO}_{2}$ film.

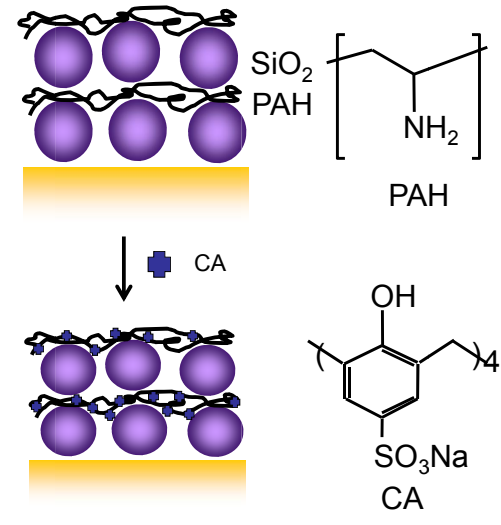

(a)

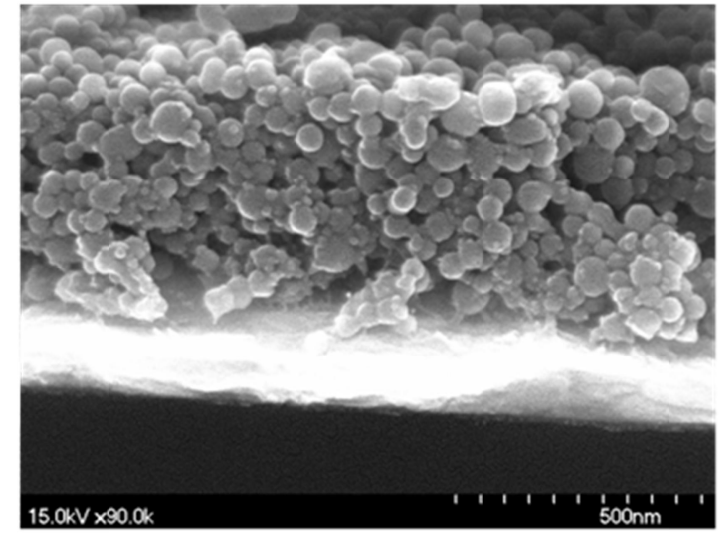

(b)

Figure 1: (a), Schematic of diagram of $\mathrm{CA}[4]$ infusion into mesoporous $\left(\mathrm{PAH} / \mathrm{SiO}_{2}\right)_{5}$ thin film deposited onto the LPG with grating period of $111.5 \mu \mathrm{m}$; (b) SEM image of the $\left(\mathrm{PAH} / \mathrm{SiO}_{2}\right)_{5}$ thin film.

After immersion into the $\mathrm{CA}[4]$ solution, the fibre was rinsed in distilled water, in order to remove physically adsorbed compounds, and dried by flushing with $\mathrm{N}_{2}$ gas. The compounds remaining in the porous silica structure were bound to the surface of the polymer layer that coated each nanosphere. This effectively increased the available surface area for the 
compounds to bond to. The presence of functional chemical compounds increased the RI of the porous coating and resulted in a significant change in the LPG's transmission spectrum, consistent with previous observations for increasing the coating thickness. All experiments have been conducted at $25^{\circ} \mathrm{C}$ and $50 \% \mathrm{of} \mathrm{rH}$.

For VOC gas measurements the LPG fibre modified with the $\left(\mathrm{PAH} / \mathrm{SiO}_{2}\right)_{5} \mathrm{CA}[4]$ film was fixed in a measurement chamber of volume $380 \mathrm{~cm}^{3}$ and constant volumes of each analyte solutions (benzene and chloroform) was placed in proximity to the sensor. The sensor response was recorded at a frequency of $1 \mathrm{~Hz}$. The transmission spectrum (TS) was recorded with each analyte solution present in the chamber and also after its removal. To regenerate the sensor response, the optical fibre was flushed using nitrogen gas.

\section{RESULTS AND DISCUSSION}

\subsection{In fusion of the functional compound}

The transmission spectrum of the LPG undergoes changes due to the alternate deposition of $\mathrm{SiO}_{2} \mathrm{NPs}$, which influences the effective RI of the cladding mode, as described previously [3]. When the LPG was in the silica colloidal solution, the resonance feature (at ca. $677 \mathrm{~nm}$ ) corresponding to coupling to the $\mathrm{LP}_{018}$ cladding mode exhibited a small linear blue wavelength shift of ca. $0.6 \mathrm{~nm} /$ layer, suggesting uniform film deposition (data not shown). As the optical thickness of the coating increased, it became possible to couple energy to the $\mathrm{LP}_{019}$ mode, with the corresponding development of the resonance band at ca. $830 \mathrm{~nm}$ [3]. In contrast to previous work [3] using this grating period the resonance feature starts to develop in air and is well-developed in water for this coating thickness.

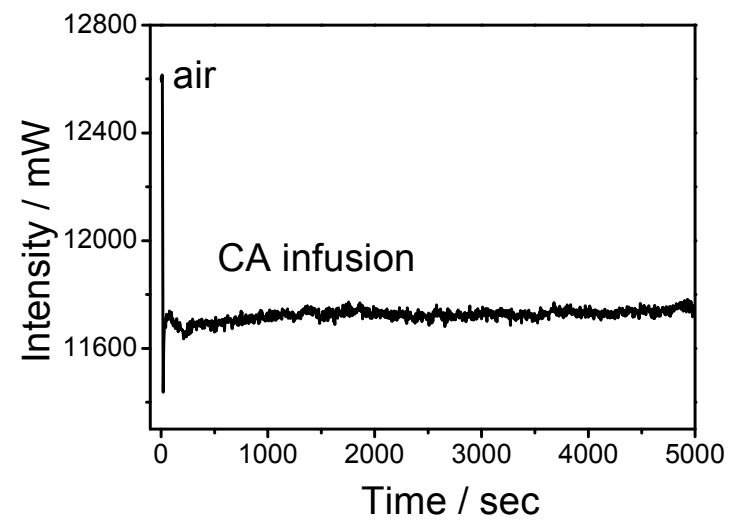

(a)

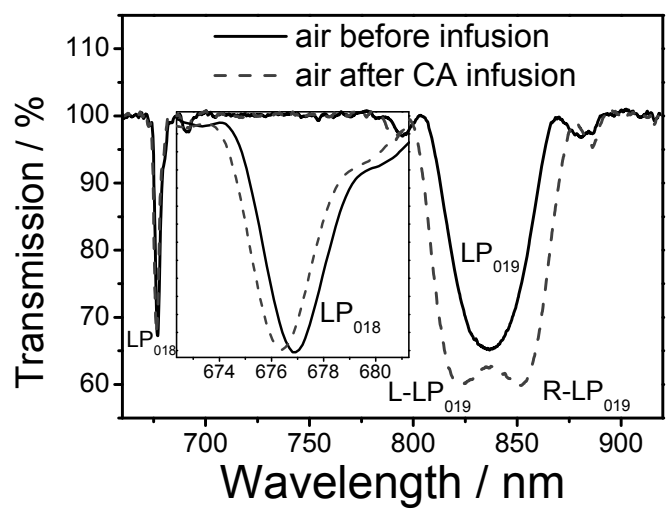

(b)

Figure 2: (a), Dynamic change in the transmission of an LPG with grating period of $111.5 \mu \mathrm{m}$ coated with the $\left(\mathrm{PAH} / \mathrm{SiO}_{2}\right)_{5}$ thin film, measured in $1 \mathrm{mM}$ aqueous solution of the CA[4] at a wavelength of $821 \mathrm{~nm}$; (b) comparison of the transmission specturm measured in air before and after infusion of the CA[4].

After deposition of the $\left(\mathrm{PAH} / \mathrm{SiO}_{2}\right)_{5}$ thin film on the LPG optical fibre it was immersed into $1 \mathrm{mM}$ aqueous solution of the CA[4] compound to provide sensor with its specificity. The small change in transmission along with the wavelength shift was observed on immersion of the LPG into functional compounds, suggesting its efficient binding in the mesopores, Figures $2 \mathrm{a}$ and $\mathrm{b}$. The sensor response saturated after ca. 15 min indicating completion of the binding process, Figure 2a. The same binding time was measured when UV-Vis spectra of the $\left(\mathrm{PAH} / \mathrm{SiO}_{2}\right)_{5}$ thin film deposited onto quartz substrates were measured (data not shown). After the LPG was withdrawn from the CA[4] solution, a blue shift of the $\mathrm{LP}_{018}$ resonance band was observed, accompanied by the splitting of the $\mathrm{LP}_{019}$ resonance band, indicating increase of the coating refractive index and thus incorporation of the $\mathrm{CA}[4]$ into the mesoporous $\left(\mathrm{PAH} / \mathrm{SiO}_{2}\right)_{5}$ thin film. In order to check its response to VOCs the LPG device was exposed to chloroform and benzene. 


\subsection{VOC response}

Figure 3a shows the dynamic sensor response measured at $821 \mathrm{~nm}$ on exposure of the coated LPG in turn to saturated concentrations of chloroform, water and benzene vapors. The transmission undergoes dramatic change after the drop of the corresponding VOC liquid is placed into the measurement chamber. The response was observed to be reversible as after the cap of the chamber was opened and the sensor was re-exposed to ambient air, i.e. at the decrease of the VOCs concentration, the transmission reverted to its previous value. The effect of relative humidity was observed to be considerably lower than the response to the VOCs when the LPG was exposed to the same amount of water, Figures 3a and $3 \mathrm{~b}$. It should be noted that when the response to water vapour was larger before the infusion of CA[4]. These results suggest that $\mathrm{CA}[4]$ provides $\left(\mathrm{PAH} / \mathrm{SiO}_{2}\right)_{5}$ film not only with the specificity to VOCs compounds, but also reduces its sensitivity to humidity, most likely owing to CA[4]'s hydrophobicity. Changes in the TS of the LPG suggest that binding of the VOCs to the CA[4] induces an increase in the RI of the mesoporous $\left(\mathrm{PAH} / \mathrm{SiO}_{2}\right)_{5} \mathrm{CA}[4]$ film, Figure $3 \mathrm{~b}$.

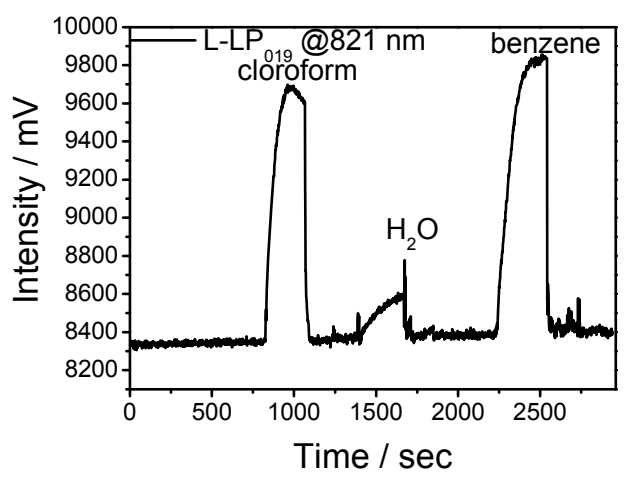

(a)

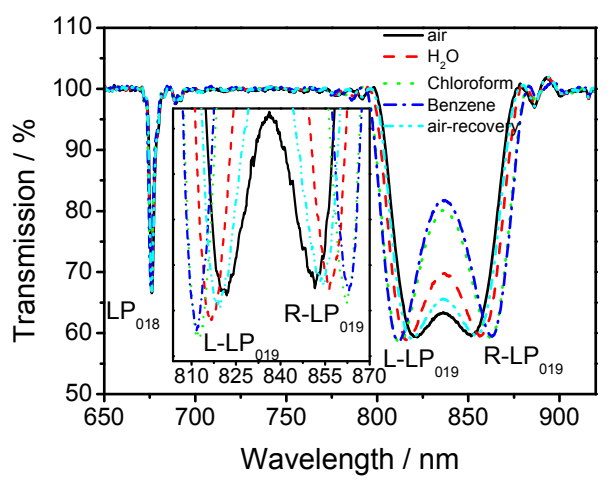

(b)

Figure 3. (a) Dynamic sensor response at the exposure to chloroform, water and benzene of the LPG with grating period of $111.5 \mu \mathrm{m}$ modified with the mesoporous $\left(\mathrm{PAH} / \mathrm{SiO}_{2}\right)_{5} \mathrm{CA}[4]$ film measured $821 \mathrm{~nm}$ (b) TS spectra measured in atmospheres of: air, chloroform, water vapour and benzene.

\section{Conclusions}

The response of the LPG sensor modified with a mesoporous $\left(\mathrm{PAH} / \mathrm{SiO}_{2}\right)_{5}$ film infused with the $\mathrm{CA}[4]$ receptor to VOCs was studied. Presence of the CA[4] receptor allows to sensitive measurements of VOCs with high reversibility and rapid response time. Future work will focus on studying the response of different VOCs and also at determining the limit of detection by measuring concentration profiles. In addition, the effect of the calixarene size on sensor performance will be investigated.

\section{References}

[1]. Hodgson, A.T., "A review and a limited comparison of methods for measuring total volatile organic compounds in indoor air," Indoor Air, 5(4), 247 (1995).

[2]. ASHRAE, "Control of gaseous indoor air contaminants", Fundamentals Handbook, Chapter 44, American Society of Heating, Refrigerating and Air Conditioning Engineers (2001).

[3].James, S.W., Tatam, R.P., "Optical fibre long-period grating sensors: characteristics and application,” Meas. Sci. Technol. 14, R49 (2003).

[4].James, S.W., Tatam, R.P., "Fibre optic sensors with nano-structured coatings" Journal of Optics A: Pure and Applied Optics. 8 (2006) S340.

[5].Korposh, S., James, S.W., Lee, S.-W, Topliss, S.M., Cheung, S.C., Batty, W.J., Tatam, R. P., "Fiber optic long period grating sensors with a nanoassembled mesoporous film of SiO2 nanoparticles." Opt. Express. 18(12), 13227 (2010).

[6].Çapan, R., Özbek, Z., Göktaş, H., Şen, S., İnce, F.G.,. Özel, M.E, A Stanciu, G., Davis, F., "Characterization of Langmuir-

Blodgett films of a calix[8] arene and sensing properties towards volatile organic vapors," Sensors and Actuators B 148, 358 (2010).

[7]. Cheung, S.C., Topliss, S.M., James S.W., Tatam, R.P., "Response of fibre optic long period gratings operating near the phase matching turning point to the deposition of nanostructured coatings," Journal of the Optical Society of America B 25, 902 (2008). 


\section{Detection of volatile organic compounds (VOCs) using an optical fibre long period grating with a calixarene anchored mesoporous thin film}

Korposh, Sergiy

SPIE

Korposh, S., Davis, F., James, S.W., Wang, T., Lee, S.-W., Higson, S. and Tatam, R.P. 2013. Detection of volatile organic compounds (VOCs) using an optical fibre long period grating with a calixarene anchored mesoporous thin film. In Jaroszewicz, L.R. (ed.) Proceedings of the 5th European workshop on optical fibre sensors, 19-22 May 2013, Krakow, Poland. Proceedings of SPIE, volume 8794, pages 131-146, paper 8794-1I. Bellingham, WA: SPIE. DOI: 10.1117/12.2026270 https://dx.doi.org/10.1117/12.2026270

Downloaded from Cranfield Library Services E-Repository 